\title{
Développement de capacités narratives des enfants bilingues: I'acquisition de l'espagnol dans le contexte suisse-allemand.
}

\section{Verónica Sánchez Abchi}

La présente contribution vise à analyser les capacités langagières d'enfants bilingues espagnol-suisse allemand à l'occasion de la production orale de récits d'expériences personnelles. En se focalisant sur la production orale en espagnol avant le début de l'école primaire, cette étude explore le développement précoce des capacités narratives considérées comme un important précurseur de l'alphabétisation initiale des enfants (Dickinson \& Tabors, 2001, entre autres). Notre étude se base sur un échantillon de textes produits par 24 enfants répartis en deux groupes d'âge, 3 et 5 ans. Nous analysons également un questionnaire soumis aux familles des enfants participants, questionnaire qui interroge les pratiques linguistiques à la maison et la biographie langagière de ces enfants. L'étude menée révèle des différences entre les deux groupes d'âge ainsi qu'un lien entre la performance discursive des enfants et les pratiques langagières familiales. Elle a en outre permis de repérer des capacités en espagnol qui potentiellement pourraient favoriser les capacités narratives dans la langue de l'entourage - le suisse-allemand, d'abord, et ensuite l'allemand standard. Enfin, ces résultats nous amènent à réfléchir aux conditions qui favorisent la conservation de la langue de la migration et ses apports potentiels à la scolarisation.

\section{Introduction}

La présente contribution, de type exploratoire, vise à analyser les capacités langagières d'enfants, amenés à devenir bilingues espagnol/suisse-allemand, à l'occasion de la production orale de récits d'expériences personnelles. L'étude est focalisée sur les capacités de production orale en espagnol, langue de la migration et minoritaire dans le contexte suisse. En se concentrant sur les productions langagières avant le début de l'école primaire, cette étude explore le développement précoce des capacités narratives considérées comme un important précurseur de l'alphabétisation initiale pour les enfants (Dickinson \& Tabors, 2001, entre autres).

Les productions narratives des enfants bilingues ont fait l'objet de nombreuses études, focalisées sur des problématiques différentes: l'expression de la tempo- 
ralité, les procédés de référence, la gestion du thème, le rôle des interactions avec les adultes et les différents inputs dans la construction de narrations (Verhoeven $\&$ Strömqvist, 2001; voir aussi la synthèse de Pavlenko, 2008). En espagnol, en revanche, les études ne sont pas si fréquentes, si on excepte celles qui concernent le contact anglais-espagnol (Gutiérrez-Clellen, 2002; Fiestas \& Peña, 2004; Ucelli \& Páez, 2007, entre autres), en particulier aux États-Unis où l'importance de la migration hispanophone met en évidence des problématiques spécifiques pour la politique éducative. Les différences repérées entre les enfants bilingues et monolingues dans ce contexte ont été souvent associées à des inégalités sociales et éducatives. En ce sens, les études ont mis en évidence le besoin d'étayer et de soutenir le développement des compétences narratives en L1, avant le début de la scolarité, pour éviter l'échec scolaire des enfants de familles migrantes (Ucelli \& Páez, 2007). Une recherche longitudinale, qui compare les capacités discursives en espagnol des enfants monolingues et bilingues basque espagnol (Almgren, Beloki, \& Manterola, 2008 ; Almgrem, Beloki, Idiazábal \& Manterola, 2008), a montré qu'à cinq ans, les enfants bilingues avaient un degré d'autonomie moindre que les monolingues. Cette différence peut s'expliquer par les difficultés de vocabulaire en espagnol éprouvées par des enfants de langue basque L1.

En fait, les études montrent que, même s'il peut y avoir une certaine variabilité dans le rythme d'acquisition entre les enfants bilingues et monolingues, les aspects les plus importants du développement linguistique sont acquis au même âge par tous les enfants si les groupes comparés sont similaires (Grosjean, 2010, pour une synthèse). Toutefois, malgré le corpus important d'études relatives au développement discursif des enfants bilingues, l'acquisition simultanée de deux voire de plusieurs langues préoccupe encore aujourd'hui, de manière fréquente, les parents concernés (Grosjean, 2013).

Notre intérêt pour les capacités linguistiques des enfants suisses ayant des racines hispanophones a émergé d'un souci actuel des parents d'enfants qui suivent des cours de langue et de culture d'origine en espagnol dans le contexte suisse alémanique ${ }^{1}$. Leurs préoccupations concernaient plusieurs aspects: a) l'acquisition simultanée de l'espagnol et du suisse-allemand, b) les progrès de leurs enfants dans l'apprentissage de l'espagnol, c) l'impact que peut avoir l'enseignement de l'espagnol sur l'acquisition de la langue de l'entourage, le suisse-allemand et, plus tard, à l'école, de l'allemand standard (Hoch-Deutsch) et, d) les difficultés à préserver l'espagnol comme langue familiale ainsi que dans le contexte élargi de la socialisation. Ces inquiétudes ont motivé une précédente étude qui tendait à explorer les capacités langagières d'enfants bilingues espagnol/ suisse-allemand de cinq ans lors de la production de récits de vie oraux. Cette étude a donc permis de mieux connaître les capacités et difficultés des enfants bilingues par rapport aux monolingues. Les résultats ont montré qu'il n'y avait pas de différences significatives dans la longueur, la complexité structurelle ou la textualisation des productions orales des enfants bilingues et monolingues. En revanche, nous avons mis en évidence des différences au niveau lexical et 
morphologique, ainsi que la présence de transferts du suisse allemand à l'espagnol au niveau syntaxique (Sánchez Abchi, 2013). Au-delà de cette expérience, il n'existe pas, à notre connaissance, d'études sur l'acquisition de l'espagnol dans le contexte suisse alémanique et moins encore d'études qui comparent les capacités narratives de ces enfants à différents moments du développement.

Étant donné que le développement des capacités narratives précoces est lié aux pratiques langagières familiales, il nous a paru intéressant de répertorier ces pratiques chez les familles des enfants bilingues espagnol-suisse allemand afin d'explorer des liens possibles avec la performance linguistique des enfants. En effet, les enfants qui ont accès en bas âge à des expériences linguistiques favorisant l'alphabétisation auront - en principe - moins de problèmes à l'école. Et cela devrait valoir aussi pour les enfants qui parlent une langue différente à l'école et à la maison (Kuyumcu \& Senyildiz, 2011). Par ailleurs, des apports d'une langue à l'autre chez les enfants bilingues peuvent être observés à plusieurs niveaux, tels que l'acquisition du système d'écriture (voir pour une synthèse Manchón, 2013) ou les caractéristiques des genres textuels (Idiazabal \& Larrigan, 1997). Dans ce processus, le niveau de maîtrise des langues joue un rôle central. À cet égard, l'évaluation des capacités narratives orales et de vocabulaire en espagnol pourrait par conséquent donner des pistes sur les potentielles contributions à la performance narrative dans une autre langue, en particulier la langue de scolarisation.

De manière générale, la question de base qui guide notre travail est la suivante: comment les enfants bilingues hispanophones, qui grandissent dans des contextes plurilingues, apprennent-ils et développent-ils la langue minoritaire, avant le début de l'école primaire?

Pour répondre à cette question, nous analysons et comparons les productions narratives d'enfants bilingues de deux groupes d'âge à 3 et 5 ans. Nous cherchons à identifier les différences entre les productions de chaque groupe, afin d'inférer des tendances dans le développement et l'acquisition de la langue minoritaire en contexte plurilingue.

Dans un deuxième temps, nous nous demandons quelles capacités langagières développées en langue maternelle pourraient être susceptibles d'être transférées à la langue de scolarisation. En nous centrant sur les productions en espagnol, notre deuxième question ne peut recevoir qu'une réponse partielle. En effet, une analyse des productions en allemand standard devrait être prise en compte pour une future comparaison et pour mettre en valeur les transferts effectifs d'une langue à l'autre. Toutefois, étant donné que nous nous centrons sur les capacités linguistiques censées contribuer au développement discursif des enfants, et, en conséquence à sa réussite scolaire, nous pourrions tirer des pistes sur les possibles contributions de l'espagnol à la langue de scolarisation.

Enfin, nous proposons une réflexion sur les pratiques langagières familiales qui favorisent le développement précoce de la langue minoritaire et qui pourraient avoir une incidence sur le processus d'alphabétisation. 


\section{Méthodologie}

\section{La population}

Pour mener cette étude, nous avons interviewé 24 enfants répartis en deux groupes. Le premier groupe (G1) était formé de 8 enfants (âge moyen: 3:5) qui assistaient au "groupe de jeu» (Spielgruppe ${ }^{2}$ ) une à deux fois par semaine. Le deuxième groupe (G2) était constitué de seize enfants (âge moyen: 5:4) fréquentant le jardin d'enfants.

Les mères de tous les enfants avaient l'espagnol comme L1 et elles étaient migrantes d'un pays hispanophone. Bien qu'il n'y ait pas d'évidence décisive en ce qui concerne la différence entre pères et mères pour la transmission de la langue (voir Thomas, 2012), nous avons pris la décision de conserver ce critère pour avoir un échantillon plus homogène et comparable.

\section{Les familles bilingues}

Un questionnaire sur les stratégies de transmission de la langue et les pratiques linguistiques de la famille, inspiré de Bregy, Brohy \& Fuchs (2000), a été fourni aux familles participantes. Les questions portaient sur a) le niveau d'instruction des parents, b) les langues parlées, c) la langue familiale, d) les formes de transmission de la langue dans la famille, d) les situations impliquant l'usage de l'espagnol, e) le choix des langues dans les habitudes socioculturelles de la famille telles que: chansons, lectures, mass-médias électroniques, f) l'importance de l'espagnol pour les parents.

Les parents des enfants forment, dans tous les cas, des couples mixtes: le père est suisse germanophone et la mère vient d'un pays d'Amérique-Latine (Argentine, Chili, Pérou, Cuba, Colombie, Équateur, République dominicaine et Mexique) ou d'Espagne.

Les résultats des questionnaires montrent que le $87 \%$ des mères et $74 \%$ des pères ont terminé des études universitaires ou tertiaires et que trois parmi eux ont suivi des études de doctorat. Le reste des parents a achevé l'école secondaire et dispose d'une formation professionnelle.

Toutes les mères sont plurilingues et parlent au moins deux langues - espagnol et allemand - et au maximum 5 langues, avec des niveaux de maîtrise différents. Toutes se disent convaincues de l'importance de transmettre et maintenir la langue espagnole, afin de conserver les liens avec le pays d'origine et la famille maternelle.

Les familles privilégient, pour la plupart, une stratégie de «dichotomie de personne» (stratégie OPOL: "one parent, one language») pour la transmission de la langue. Dans certains cas, les familles établissent une dichotomie de «lieu», en parlant une langue dans le contexte familial et la langue de l'entourage dans les autres contextes (Schmidt-Mackey, 1977). Finalement, en ce qui concerne les actions pour la conservation de la langue, les familles mettent en pratique des stratégies diverses pour l'apprentissage de l'espagnol, telles que la lecture 
fréquente de contes, le contact avec des chansons et des films en espagnol, la communication fréquente avec la famille de la mère dans le pays d'origine. Dans un cas, la mère a également parlé de la mise en place des stratégies d'alphabétisation spécifiques en espagnol (découverte des lettres et écriture de mots).

\section{Les entretiens avec les enfants}

Lors de l'entretien avec les enfants, nous leur avons demandé de raconter un récit de vie, sur le thème de la «blessure». Afin d'introduire le sujet, la chercheuse commençait l'entretien par un récit de vie personnel, puis elle demandait à l'enfant s'il s'était déjà fait mal. Nous avons enregistré et transcrit les entretiens.

Nous avons choisi de focaliser notre étude sur l'appropriation du genre «récit de vie», parce qu'il s'agit d'un genre textuel très fréquent au début de la scolarité et dans la vie quotidienne. Il est, par conséquent, assez familier pour les enfants. Il s'agit aussi d'un genre fréquent dans les recherches sur le bilinguisme (Pavlenko, 2008).

\section{Procédure d'analyse des productions}

Pour l'analyse, nous prendrons en considération la planification de texte et la linéarisation ou textualisation qui intègre les différents aspects linguistiques liés à la production (Schneuwly \& Dolz, 2004). La planification de la production des enfants sera examinée en fonction des caractéristiques du genre textuel - le récit de vie - et nous nous servirons des catégories de Labov (1972) pour la description de l'organisation textuelle: résumé, orientation, complication et résolution ${ }^{3}$. Concernant la textualisation, les mécanismes de cohésion nominale et verbale ainsi que l'emploi des organisateurs textuels seront analysés (Fayol \& Schneuwly, 1987). On examinera aussi les aspects transversaux dans la production textuelle (Dolz, Gagnon, Mosquera \& Sánchez Abchi, 2013): la syntaxe - construction et relation entre les composantes de la phrase -, la morphologie et le niveau lexical. Pour estimer l'autonomie narrative, nous avons élaboré un indice que nous définissons en calculant la moyenne des mots par intervention de l'adulte. Cet indice nous permet de pondérer le poids du soutien de l'adulte pour faire avancer le récit. La longueur des textes sera aussi considérée.

\section{Résultats}

Une analyse préliminaire des données permet de constater des différences dans les productions des enfants. Ces différences concernent toutes les catégories d'analyse: la longueur, la planification et les phénomènes de textualisation de la production, ainsi que le niveau d'autonomie narrative des enfants.

D'abord, nous avons comparé la longueur des textes en considérant le nombre de mots dans chaque production. Le tableau 1 synthétise les données. Les chiffres indiquent la moyenne (M) et l'écart type (ET) pour chaque groupe. 
Tableau 1. Longueur des textes. Moyenne et écart type

\begin{tabular}{|l|l|}
\hline 3 ans & 5 ans \\
\hline $\mathrm{M}=29,5$ & $\mathrm{M}=55,2$ \\
$(\mathrm{ET}=12,16)$ & $(\mathrm{ET}=27,9)$ \\
\hline
\end{tabular}

Nous remarquons que les productions des enfants de 5 ans (G2) sont plus longues que celles des enfants de 3 ans (G1). Bien que la différence soit significative ( $T=2.490 ; p=0.021)$, l'écart type reste élevé, ce qui suggère une variation importante à l'intérieur de chaque groupe. Dans ce sens, on peut interpréter les résultats simplement comme une tendance.

En ce qui concerne le niveau de planification de la production, on a constaté l'adéquation des productions au genre "expérience personnelle», en prenant en compte les catégories de Labov (1972). Le texte ci-dessous exemplifie les catégories trouvées dans les narrations des enfants:

E: ¿̨Y vos alguna vez te lastimaste?

$N$ : En un viaje estaba ahi con mi mama y yo viaje en una loma peligrosa donde bajan tractores con mucha piedra y estaba sangrando aqui y aqui y aqui y toda la parte... tanta sangre me dolió.

$\mathrm{E}:$ ¿Y eras muy chiquita?

N: Todavía iba a Spielgruppe

Enfant bilingue, 5 ans (E: intervieweuse; $\mathrm{N}$ : prénom enfant)

[E: (...) Et toi, tu t'es fait mal?

$N$ : Pendant un voyage j'étais avec ma mère et j'ai voyagé dans une colline dangereuse où les tracteurs descendent avec beaucoup de pierres et j'ai saigné ici, et ici et ici et toute la partie ... aussi de sang et ça m'a fait mal.

E: Et tu étais petite?

$N$ : J'allais encore au Spielgroupe $]^{4}$

Dans l'exemple, il y a une absence de la catégorie résumé, car l'enfant commence directement avec l'orientation: «pendant un voyage, j'étais avec ma mère et j'ai voyagé dans une colline dangereuse..." La complication n'est pas complète, mais ses conséquences sont explicites: “j’ai saigné...» et on peut comprendre que l'enfant est tombée dans cette «colline dangereuse». Le texte n'a pas de résolution.

La planification des textes met en évidence des différences dans les productions des deux groupes d'âge. Le tableau 2 illustre les catégories présentes dans les textes des enfants. Les chiffres indiquent pour chaque catégorie le pourcentage de textes qui les prennent en compte. 
Tablean 2. Pourcentage de présence de catégories

\begin{tabular}{|l|l|l|}
\hline & $\begin{array}{l}\text { G1 } \\
3 \text { ans }\end{array}$ & $\begin{array}{l}\text { G2 } \\
5 \text { ans }\end{array}$ \\
\hline Résumé & $62 \%$ & $53 \%$ \\
\hline Orientation & $50 \%$ & $87 \%$ \\
\hline Complication & $100 \%$ & $100 \%$ \\
\hline Résolution & $75 \%$ & $60 \%$ \\
\hline
\end{tabular}

Les narrations se centrent surtout sur la complication, reprise par la totalité des enfants. Mais l'orientation, qui permet de contextualiser l'action, est clairement plus fréquente chez les enfants plus âgés, ce qui est comparable aux résultats trouvés chez les enfants monolingues (voir Borzone, 2003, pour une synthèse). Par contre, les enfants plus jeunes tendent à présenter seulement le résumé plus fréquent dans le G1 - et développent leurs récits à partir du dialogue avec l'adulte.

La résolution est moins présente dans les textes du G2, ce qui peut être surprenant. Une explication possible serait que les enfants de 3 ans (G1) intègrent la conclusion grâce aux questions de l'intervieweuse. En revanche, les enfants qui narrent de manière plus autonome finissent leurs récits après avoir développé l'orientation et la complication.

En effet, l'étayage de l'adulte et l'interaction se révèlent être des facteurs importants pour la production. Le fragment ci-dessous met en évidence la manière dont l'enfant se sert des questions de l'adulte pour faire avancer son récit:

E: ¿Estabas andando en patineta?

J: Y frenó y hace una curva

E: ¿Pero qué te hiciste?

J: Me duele pero ya no me duele

E: ¿Te hiciste daño?

J: Si y ya me cur... pero hasta casa se me curó

Ah, se te curó en el camino a casa. Y dónde te hiciste daño?

J: Aqui, en la frente

$\mathrm{E}:$ ¿Y quién te ayudó?

J: Mamá

E: ¿Te puso una tirita?

$\mathrm{J}$ : No, porque ya no... porque ese era el susto

E: Ah. Era más un susto que otra cosa. Sos un chico con suerte.

Enfant bilingue 3 ans (E: intervieweuse; J: prénom enfant)

[E: Tu allais avec la trottinette? 
J: Oui (il) a freiné et (il) et (il) tourne.

E: Mais qu'est que tu as fait?

J: Ça me fait mal, mais il ne me fait plus mal

$\mathrm{E}: \mathrm{Tu}$ t'es fait mal ?

J: Oui, mais c'est déjà bien. Jusqu'à la maison, c'est déjà bien.

$\mathrm{E}$ : Ah, en allant à la maison, tu t'es senti mieux. Et tu t'es fait mal où ?

J: Ici, sur le front

E: Qui t'a aidé ?

J: Mama

E: Elle t'a mis un pansement?

J: Non, parce que .. Parce que j'avais eu peur.

E: Ah... c'était plutôt la peur. Tu es un enfant chanceux!]

Pour mieux estimer le niveau d'autonomie narrative des enfants, nous avons calculé la moyenne des mots par nombre d'interventions de l'adulte dans chaque groupe. Dans le G1, la moyenne est de 4,25 (ET=2,88), tandis qu'elle est notablement plus élevée dans le groupe des enfants plus âgés: 8,1(ET=3,34). L'indice permet d'observer que les enfants les plus âgés deviennent plus «indépendants» du soutien de l'adulte pour construire leur production. Autrement dit, la tendance montre que les enfants passent, progressivement, d'une planification fortement soutenue par l'adulte à une planification plus autonome.

En ce qui concerne la textualisation, nous avons considéré quatre aspects principaux: la cohésion nominale et verbale, la moyenne d'emploi des organisateurs textuels et la moyenne de constructions subordonnées. Le tableau 3 résume les observations de l'analyse.

Tableau 3. Niveau de textualisation dans les productions des enfants

\begin{tabular}{|l|l|l|}
\hline Textualisation & $\begin{array}{l}\mathrm{G} 1 \\
\text { Enfants 3 ans }\end{array}$ & $\begin{array}{l}\mathrm{G} 2 \\
\text { Enfants 5 ans }\end{array}$ \\
\hline Cohésion nominale & Adéquate & Adéquate \\
\hline Cohésion verbale & $\begin{array}{l}\text { Adéquate alternance Parfait } 5 / \\
\text { Imparfait } \\
\text { (un cas de mélange présent/ passé) }\end{array}$ & $\begin{array}{l}\text { Adéquate alternance Parfait } \\
\text { Imparfait }\end{array}$ \\
\hline Organisateurs textuels & $\mathrm{M}=2,37(\mathrm{ET}=2,06)$ & $\mathrm{M}=4,93(\mathrm{ET}=3,25)$ \\
\hline Constructions subordonnées & $\mathrm{M}=0,25$ & $\mathrm{M}=1.8$ \\
\hline
\end{tabular}

Quant à la cohésion nominale, les reprises nominales sont adéquates, centrées sur la première personne. Il faut remarquer que les possibles problèmes d'ambiguïté sont résolus grâce au contexte. De même, les productions de tous les enfants respectent la cohésion verbale et l'opposition parfait/imparfait - typique du discours narratif, est bien maitrisée. La seule exception est un cas dans le G2, dans lequel l'enfant utilise parfois l'imparfait de manière indistincte: "tenía que ... [je devais... ]» au lieu de "tuve que...[j'ai dî...]». 
Il faut remarquer que la distinction entre les temps du passé n'existe pas en suisse-allemand. L'emploi de temps de verbes en espagnol pourrait donc être affecté par la langue de l'entourage.

Une différence importante entre les deux groupes concerne les organisateurs textuels. En effet, les enfants les plus âgés emploient plus du double d'organisateurs dans leurs textes, et avec une plus grande diversité. Cet aspect suggère une avance dans le niveau de vocabulaire de ce domaine. De plus, comme les organisateurs aident à mieux structurer l'information, cette différence permet d'inférer un progrès dans les capacités narratives des enfants.

D'ailleurs, la moyenne de constructions subordonnées, considérée comme un indice de la complexité syntaxique des textes (Hunt, 1970 ; Véliz, 1988, pour l'espagnol), est aussi plus élevée chez les enfants de 5 ans. Cette différence, qui peut être un indice purement développemental également observable chez les enfants monolingues (voir synthèse de Silva, 2008, pour l'espagnol), relève aussi d'une avance dans la capacité narrative. En effet, les phrases subordonnées sont souvent employées pour donner des précisions temporelles ou spatiales et pour se référer au deuxième plan narratif (Berman, 1999; Berman \& Slobin, 1994). L'exemple déjà présenté illustre cette fonction:

«Una loma peligrosa, donde bajan tractores con mucha piedra» (enfant bilingüe, 5 ans)

[une colline dangereuse, où les tracteurs descendent avec beaucoup de pierres]

Au-delà de la complexité syntaxique - par ailleurs discutée (Silva, 2008), l'emploi de subordonnées chez les enfants du G2 semble être en rapport avec la catégorie «orientation» et est donc lié à l'enrichissement de la structure narrative.

Aux niveaux syntaxique, lexical et morphologique, pour pouvoir comparer les deux groupes, nous avons répertorié et classé des phénomènes particuliers. Le tableau 4, ci-dessous, synthétise les phénomènes repérés et montre leur fréquence dans les textes. Les chiffres indiquent la quantité de textes comprenant chaque observation. 
Tableau 4. Phénomènes de textualisation aux niveaux syntaxique, morphologique et lexical

\begin{tabular}{|c|c|c|c|c|}
\hline & \multicolumn{2}{|l|}{ Phénomènes } & Fréq $3 \mathrm{~A}$ & Fréq $5 \mathrm{~A}$ \\
\hline \multirow[t]{3}{*}{$\begin{array}{l}\text { Niveau } \\
\text { syntaxique }\end{array}$} & \multicolumn{2}{|c|}{$\begin{array}{l}\text { Répétition du pronom sujet de la première personne, } \\
\text { qui peut être omis en espagnol: } \\
\text { "Yo" ("Je») "yo viajé en una loma peligrosa». }\end{array}$} & 2 & 9 \\
\hline & \multicolumn{2}{|c|}{$\begin{array}{l}\text { Ordre syntaxique typique du suisse-allemand: verbe de } \\
\text { la construction subordonnée à la fin de la phrase: } \\
\text { yo manejé muy rápido «hasta que nada vi» au lieu de } \\
\text { «hasta que no vi nada» } \\
\text { [j'allais aussi vite que je n'ai rien vu] }\end{array}$} & 1 & 1 \\
\hline & \multicolumn{2}{|c|}{$\begin{array}{l}\text { Rection } \\
\text { Absence d'objet direct, requis par le verbe: «Fui a coser» } \\
\text { [Je suis allé coudre] } \\
\text { Utilisation inadéquate des prépositions }\end{array}$} & 1 & 3 \\
\hline \multirow[t]{2}{*}{$\begin{array}{l}\text { Niveau } \\
\text { morphologique }\end{array}$} & \multicolumn{2}{|c|}{$\begin{array}{l}\text { Formes verbales } \\
- \text { Me «cayó» ( } 3^{\mathrm{e}} \text { personne) au lieu de «me caí» }\left(1^{\mathrm{e}}\right. \\
\text { personne) [J'est tombée, au lieu de Je suis tombée] }\end{array}$} & 2 & 3 \\
\hline & \multicolumn{2}{|c|}{$\begin{array}{l}\text { - Régularisation des verbes irréguliers «poní » au lieu de } \\
\text { "puse» ("J'ai mis») }\end{array}$} & 3 & 2 \\
\hline \multirow[t]{6}{*}{ Niveau lexical } & \multicolumn{2}{|c|}{ Inclusion de mots en suisse-allemand } & 1 & 11 \\
\hline & \multicolumn{2}{|c|}{$\begin{array}{l}\text { Confusion de termes de la même famille: «Tengo ojos } \\
\text { muy bien» au lieu de «muy buenos» } \\
\text { ["J'ai des yeux très bien» au lieu de «très bons»] }\end{array}$} & 0 & 1 \\
\hline & \multirow[t]{4}{*}{$\begin{array}{l}\text { Emploi de stratégies } \\
\text { discursives }\end{array}$} & $\begin{array}{l}\text { Onomatopée } \\
\text { "Y me hice Push» [je me suis } \\
\text { fait Push] }\end{array}$ & 0 & 5 \\
\hline & & $\begin{array}{l}\text { Déictiques: } \\
\text { "Me lastimé acá» } \\
\text { [je me suis fait mal ici] }\end{array}$ & $\begin{array}{l}8 \text { (surtout } \\
\text { pour le } \\
\text { corps) }\end{array}$ & 7 \\
\hline & & $\begin{array}{l}\text { Paraphrase explicative: } \\
\text { «Sabés, con eso que pica fuerte» } \\
\text { [Tu sais, avec ça qui pique fort] }\end{array}$ & 0 & 1 \\
\hline & & Mot général: «coso» [truc] & 0 & 1 \\
\hline
\end{tabular}

La régularisation des verbes et l'emploi de mots généraux sont des phénomènes également très fréquents chez les enfants monolingues de 3 et 5 ans. Les autres constructions peuvent aussi être observées dans les productions d'un locuteur qui apprend l'espagnol comme L2.

Il vaut la peine de remarquer, toutefois, les différences les plus importantes entre les deux groupes. Celles-ci se concentrent sur le domaine lexical et, pour la syntaxe, sur la répétition du pronom sujet. En effet, dans une langue pro-drop ${ }^{6}$, comme l'espagnol, la répétition du pronom sujet n'est pas nécessaire, mais elle est obligatoire en suisse-allemand. La fréquence de ce phénomène augmente 
clairement chez les enfants les plus âgés. En ce qui concerne le lexique, la présence de mots en suisse-allemand est beaucoup plus faible dans le groupe d'enfants de 3 ans et sa fréquence augmente fortement dans les productions du G2. Il est important de remarquer que certains mots employés désignent des éléments spécifiques du contexte de socialisation de l'enfant, comme "kita» (crèche), «Spielplatz» (aire de jeu) ou même «Trottinette» (mot français utilisé dans le contexte bernois). Ces mots en particulier sont aussi employés par les mères hispanophones, avec les déterminants en espagnol («el Trottinette», «la Spielplatz»). Dans ce cas en particulier, l'emploi de ces alternances relève plutôt d'un code switching lié aux mots qui relèvent de la culture locale. D'un autre côté, les enfants utilisent des stratégies discursives, pour remplacer les mots qu'ils ne trouvent pas en espagnol. Les stratégies identifiées sont: les déictiques, les onomatopées, les paraphrases explicatives d'un mot qu'ils ne connaissent pas et l'usage d'un mot général: «coso/cosa » (le truc).

Les enfants de 3 ans utilisent surtout les déictiques, tandis que, dans le G2, on observe aussi les autres ressources. En ce sens, il est possible d'inférer un développement progressif des stratégies discursives lié au développement de la compétence narrative.

\section{Discussion et conclusion}

L'analyse des données nous montre qu'il y a des différences entre les deux groupes et que ces différences relèvent à la fois d'aspects du développement en général et d'aspects spécifiques des situations de contact entre les langues. En ce qui concerne la longueur des textes, les productions des enfants de 5 ans sont plus longues, en quantité de mots. Au niveau de la planification, les textes de ces enfants donnent plus de précisions concernant le contexte et la situation communicative, informations comprises dans la catégorie "orientation». Si on compare ces textes avec les productions des enfants plus jeunes, on constate que le progrès observé dans la construction des récits de vie est comparable à l'avance observée chez les enfants monolingues avec des conditions d'expérimentation similaires (Plana, 2011).

Un deuxième point à remarquer est l'augmentation de l'autonomie narrative chez les enfants les plus âgés par rapport au G1. En même temps, dans les productions du G2, on a observé une présence plus forte de mots en suisse-allemand, souvent interprétée comme une difficulté à trouver le mot en espagnol. En effet, à la lumière de travaux antérieurs (Almgren, Beloki, \& Manterola, 2008), on pourrait penser que l'autonomie narrative, liée, d'une certaine manière, à l'accès lexical en espagnol, pouvait être touchée par les difficultés lexicales. En revanche, on observe que les enfants se servent de mots en suisseallemand pour faire progresser leurs textes. Dans l'interaction, également, ils font appel à des stratégies discursives, telles que les onomatopées, les explications, les 
mots généraux, les déictiques et les gestes. Ces stratégies deviennent donc à la fois une ressource pour éviter les difficultés lexicales mais aussi pour enrichir le récit et attirer l'attention de l'interlocuteur. Dans ce sens, on peut déduire que les enfants les plus âgés font preuve d'une certaine maturation de leurs capacités pour narrer leurs expériences.

Par ailleurs, comme nous l'avons vu dans l'analyse, l'emploi de certains termes en suisse-allemand est en lien étroit avec les contextes de socialisation des enfants. Autrement dit, ces mots empruntés à la langue du contexte ne mettent pas en évidence une difficulté mais plutôt le biculturalisme de l'enfant qui communique en espagnol à la maison mais qui va à "la kita» et au «Spielplatz» pour rencontrer ses copains.

D'un autre côté, il faut aussi considérer de manière particulière la contrainte de la mémoire et de la langue dans laquelle l'événement a été "vécu». En effet, les études montrent que les expériences sont mieux récupérées en mémoire si la langue utilisée est la même langue que celle dans laquelle l'expérience a eu lieu (Marian \& Neisser, 2000). Dans le cas qui nous occupe, si l'expérience que les enfants relatent a eu lieu à la crèche ou sur l'aire de jeu, ils doivent récupérer l'information en mémoire, l'organiser et, de plus, la traduire en espagnol. Cela suppose une situation de surcharge supplémentaire qui peut aussi avoir un impact sur la sélection lexicale.

Cela dit, il est vrai que l'augmentation, dans les productions des enfants du G2, des éléments lexicaux de la langue de l'entourage, de même que la présence plus fréquente du sujet - obligatoire en suisse-allemand, peut être interprétée comme une conséquence de l'importance que prend, progressivement, le suisse-allemand pour les enfants.

En effet, le niveau de développement langagier est en rapport avec plusieurs facteurs; parmi eux, la quantité et le type d'input, le rôle de la famille, de l'école et de la communauté, les attitudes envers la langue, la culture et le bilinguisme et, finalement, le besoin de parler une langue (Grosjean, 2010). Dès que les enfants commencent à aller à l'école, même si l'input en espagnol reste stable, leurs besoins de communiquer en suisse-allemand et en allemand standard s'élèvent considérablement et cela a - ou peut avoir - un effet sur la maîtrise de l'espagnol.

Dans un autre ordre d'idée, le niveau de développement discursif devrait être mis en relation avec les pratiques linguistiques des familles. En effet, la conservation de la langue espagnole suppose un effort important. Toutes les familles participantes de cette étude avaient mis en place des stratégies pour que l'espagnol soit vraiment utilisé: films, chansons, contes, en espagnol, et une volonté explicite de parler et faire parler les enfants dans la langue minoritaire. Le développement langagier en espagnol ne va pas de soi et il peut se révéler comme un vrai défi.

Dès lors, cette difficulté s'est révélée également dans la constitution d'un échantillon pour l'étude; trouver des participants a exigé un travail de recherche, entravé par diverses difficultés. Dans certaines familles, en effet, c'est le père qui 
est hispanophone et le temps d'interaction en espagnol avec les enfants ne semble pas suffisant pour promouvoir le développement actif de la langue: les enfants peuvent comprendre la langue, mais ne la parlent pas (au moins, pas aux âges compris pour cette étude). Dans d'autres familles, seul l'aîné parle l'espagnol, mais les interactions entre frères et sœurs se développent dans la langue majoritaire - situation assez fréquente, par ailleurs, dans les familles mixtes (BarronHauwaert, 2011).

Les enfants interviewés pour cette étude écoutaient de manière fréquente des contes en espagnol et interagissaient activement dans cette langue avec leurs mères et avec d'autres hispanophones. Les enfants étaient donc exposés à des modèles narratifs dans la langue maternelle.

La maitrise de la production narrative en espagnol revêt une double importance pour la scolarisation de ces enfants. D'un côté, comme nous l'avons déjà dit plus haut, le développement précoce de la capacité narrative est considéré comme un précurseur important de l'alphabétisation initiale. Dans l'interaction avec l'adulte, l'enfant construit le récit et il apprend, en même temps, à sélectionner l'information pertinente, à organiser son récit et à mettre en place des ressources pour attirer l'attention de l'interlocuteur. De plus, en écoutant des contes et des histoires, il découvre le style de la langue écrite, dimension fondamentale pour l'apprentissage de l'écriture (Borzone, 2003). Ces capacités peuvent éventuellement apporter des contributions aux autres langues parlées par l'enfant (Ucelli \& Páez, 2007). À cet égard, les acquis dans la langue familiale peuvent être positifs pour la langue de scolarisation. Des recherches futures devraient pouvoir estimer plus précisément la nature de l'interaction entre l'espagnol et le suisse-allemand en contexte scolaire.

Un deuxième atout concerne l'apport de l'hétérogénéité et la richesse de pouvoir articuler et comparer les connaissances issues de langues différentes, en contexte scolaire. Cette capacité, dont le développement est à la base des approches d'éducation plurilingue (De Pietro, 2008), peut être récupérée pour les apprentissages seulement si l'école est prête à mettre en valeur les langues de la migration présentes dans la classe. Cette problématique ouvre, pourtant, un autre débat qui dépasse l'objet de cette contribution.

Les observations qui découlent de cette étude, bien qu'exploratoire, nous permettent d'écarter les soucis des parents, à l'origine de ce travail. Les obstacles que l'espagnol pourrait entraîner pour l'apprentissage de l'allemand ne semblent pas réalistes, si l'on considère que la langue majoritaire prend progressivement plus de place et que cela se manifeste aussi dans les productions des enfants plus âgés. Dans tous les cas, les pratiques d'alphabétisation au sein de la famille peuvent avoir une incidence positive sur la performance des enfants, qu'ils soient migrants ou non (Kuyumcu \& Senyildiz, 2011).

De même, l'école représente un défi pour la conservation de l'espagnol. En ce sens, un travail conscient et constant pour maintenir vivante la langue minoritaire s'avère nécessaire: pour pouvoir conserver la langue espagnole, il semble 
important de mettre en place, dès le début de la scolarité, des stratégies visant à créer la nécessité de parler et d'utiliser la langue minoritaire dans la famille. Ce travail demande, également, une conscience et une mise en valeur du capital linguistique des enfants bilingues et de son potentiel apport à l'apprentissage de la langue de scolarisation.

\section{Notes}

1 Les cours de langue et de culture d'origine (LCO) sont organisés par la communauté issue de la migration et sont destinés à tout enfant qui parle la langue d'origine en question dans sa famille. Conférence suisse des directeurs cantonaux de l'instruction publique. [http://www.edk.ch/dyn/19256.php]. Dans notre cas, il s'agit de l'école latino-américaine de Berne.

2 Le «Spielgrouppe» est un espace d'éducation informel pour les enfants en bas âge, avant le jardin d'enfants. Les enfants jouent en petites groupes, sous la tutelle d'un adulte. Le temps et la fréquence de chaque rencontre peut varier d'un groupe à l'autre.

3 Nous nous servons des travaux de Labov, parce que ses études sur les productions narratives d'expériences personnelles ont porté sur différents groupes d'âge (adultes, adolescents et même enfants) et que la structure identifiée nous permet d'opérationnaliser l'analyse de la planification discursive. Nous avons simplifié les catégories de Labov pour les adapter à notre corpus en nous inspirant de travaux sur les récits de vie des enfants, en espagnol (Borzone, 2003). Nous avons donc exclu les catégories «évaluation» et «coda».

4 Les mots en gras indiquent que les expressions ne sont pas adéquates en espagnol.

5 Il s'agit du "perfecto simple» ou "perfecto compuesto», temps verbaux de l'espagnol, qui pourraient, dans certains cas, correspondre au passé composé du français.

6 Le pronom sujet peut être omis.

\section{Bibliographie}

Almgren, M., Beloki, L., \& Manterola, I. (2008). The acquisition of narrative skills by Spanish L1 and L2 speakers. In J. Bruhn de Garavito \& E. Valenzuela (Éd.), Selected Proceedings of the 10th Hispanic Linguistics Symposium (pp. 146-156) Omerville, MA: Cascadilla Proceedings Project.

Almgren, M., Beloki, L., Idiazábal, I., \& Manterola, I. (2008). Acquisition of Basque in successive bilinguism. Data from oral story telling. In P. Siemund \& N. Kintanta (Éd.), Language contact and contact languages (pp. 239-259). Hamburg: John Benjamins Publishing Company.

Barron-Hauwaert, S. (2011). Bilingual Siblings. Language Use in Families. Toronto: Multilingual Matters.

Berman, R.A. (1999). Bilingual proficiency/proficient bilingualism in Hebrew-English narrative texts. In G. Extra \& L. Verhoeven (Éd.), Language Change in Migration Contexts (pp. 187-218). Berlin: Mouton de Gruyter.

Berman, R. \& Slobin, D. (1994). Relating Events in Narrative: A Crosslinguistic Developmental Study. Hillsdale, NJ: Lawrence Erlbaum Associates.

Borzone, A. (2003). Leer y escribir a los 5. Buenos Aires: Aique.

Bregy, A.-L., Brohy, C., \& Fuchs, G. (2000). Expérience d'apprentissage bilingue précoce: résultats des élèves de Monthey et de Sion à la fin de la 2e année primaire: année 1998/99. Neuchâtel: Institut de recherche et de documentation pédagogique (IRDP).

De Pietro, J.-F. (2008). Multiculturalité: maîtrise de la langue locale et ouverture à la diversité. Enjeux pédagogiques, 8, 16-19.

Dickinson D., \& Tabors P. (Éd.). (2001). Beginning literacy with language: Young children learning at home and school. Baltimore: Brookes. 
Dolz, J., Gagnon, R., Mosquera, S., \& Sánchez Abchi, V. (2013). Producción escrita y dificultades de aprendizaje. Barcelona: Graó.

Fayol, M., \& Schneuwly, B. (1987). La mise en texte et ses problèmes. In J.-L. Chiss, J.-P. Laurent, J.C. Meyer, H. Romian \& B. Schneuwly (Éd.), Apprendrelenseigner à produire des textes écrits (pp. 223-239). Bruxelles: Duculot.

Fiestas, C., \& Peña, E. (2004). Narrative Discourse in Bilingual Children Language and Task Effects. Language, Speech, and Hearing Services in Schools, 35, 155-168.

Grosjean, F. (2010). Bilingual: Life and Reality. Cambridge, Mass: Harvard University Press.

Grosjean, F. (2013). Speech production. Chapter 3. In F. Grosjean \& P. Li (Éd.), The Psycholinguistics of Bilingualism (pp. 50-69). Malden, MA \& Oxford: Wiley-Blackwell.

Gutiérrez-Clellen, V. (2002). Narratives in Two Languages: Assessing Performance of Bilingual Children. Linguistics and Education, 13(2), 175-197.

Hunt, K.W. (1970). Recent measures in syntactic development. In M. Lester (Ed.), Reading in applied transformational grammar (pp. 179-192). Nueva York: Holt, Rinehart and Wiston.

Idiazabal, I., \& Larrigan, L.M. (1997). Appropriation des langues en situation de contact. Transfert de maîtrises discursives dans un programme d'enseignement bilingue basque-espagnol. Acquisition et interaction en langue étrangère 10. [En ligne], mis en ligne le 10 octobre 2005, consulté le 20 avril 2014. URL: http://aile.revues.org/1319

Kuyumcu, R., \& Senyldiz, A. (2011). Familale Literalitätserfahrungen türkisch und russischsprachiger Kindergartenkinder. Schweizer Zeitschrift für Bildungswissenschaften, 33(1), 109-124.

Labov, W. (1972). Sociolinguistic Patterns. Philadelphia: University of Pennsylvania Press. Manchón, R.M. (2013). Writing. In F. Grosjean \& P. Li (Éd.), The Psycholinguistics of Bilingualism (pp. 100-115). Malden, MA \& Oxford: Wiley-Blackwell.

Marian, V., \& Neisser, U. (2000). Language-dependent recall of autobiographical memories. Journal of Experimental Psychology: General, 129, 361-368.

Pavlenko, A. (2008). Narrative analysis. In L. Wei \& G. Moyer (Éd.), The Blackwell guide to research methods in bilingualism and multilingualism (pp. 311-325). Oxford: Blackwell Publishing.

Plana, M.D. (2011). Los niños en un mundo de eventos: representaciones mentales y relatos. Tesis de Doctorado en Ciencias del Lenguaje, Universidad Nacional de Córdoba.

Sánchez Abchi, V. (2013). Desarrollo discursivo en contexto multilingües: Un estudio contrastivo de niños bilingües y monolingües. Revista Lengua y Habla, 17, 199-210.

Schmidt-Mackey, I. (1977). Language Strategies of the bilingual familiy. In W. Mackey \& T. Andersson (Éd.), Bilingualism in early childhood (pp. 132-143). Massachusetts: Newbury House publishers/Rowley.

Schneuwly, B., \& Dolz, J. (2004). Gêneros orais e escritos na escola. Sao Paulo: Mercado de Letras.

Silva, M.L. (2008). Desarrollo y gramaticalización de las Cláusulas relativas en el discurso infantil: aportes desde el enfoque cognitivo-prototípico. Tesis doctoral. La Plata: Univ. Nacional de La Plata, Argentina.

Thomas, C. (2012). Growing Up with Languages: Reflections on Multilingual Childhoods. Bristol: Multilingual Matters.

Uccelli, P., \& Páez, M.M. (2007). Narrative and vocabulary development of bilingual children from kindergarten to first grade: Developmental changes and associations among English and Spanish skills. Language, Speech, and Hearing Services in Schools, 38(3), 225-236.

Véliz, M. (1988). Evaluación de la Madurez Sintáctica en el discurso escrito. RLA: Revista de lingüistica teórica y aplicada, 26, 105-140.

Verhoeven, L., \& Strömqvist, S. (Éd.). (2001). Narrative development in a multilingual context (Vol. 23). Hillsdale, NJ: John Benjamins Publishing. 
Mots-clés: Bilinguisme, capacités narratives, acquisition de l'espagnol, langue minoritaire.

\section{Die Entwicklung narrativer Kompetenzen zweisprachiger Kinder: Der Erwerb der spanischen Sprache im deutschsprachigen Kontext}

\section{Zusammenfassung}

Der folgende Artikel analysiert die sprachproduktiven Fähigkeiten von zweisprachigen

Kindern (spanisch und schweizerdeutsch) bei der Wiedergabe persönlicher Erfahrungen. Die Studie fokussiert auf die mündliche Sprachproduktion von Spanisch vor Beginn der Primarschule und untersucht die Entwicklung narrativer Kompetenz als wichtigem Faktor für die sogenannte initiale Alphabetisierung (Dickinson \& Tabors, 2001, u.a.). Die Studie basiert auf einer Stichprobe mit Texten von 24 Kindern zwischen drei und fünf Jahren. Die Kinder wurden in zwei Altersgruppen eingeteilt. Ebenfalls analysiert wurden die Antworten von Eltern der teilnehmenden Kinder auf Fragen zu sprachlicher Praxis und Sprachbiografie ihrer Kinder. Neben Unterschieden zwischen den beiden Altersgruppen zeigte sich ein Zusammenhang zwischen der diskursiven Leistung der Kinder und der Praxis der Sprachen in der Familie. Ebenso erwiesen sich sprachliche Fähigkeiten auf Spanisch als potentieller Vorteil für die narrativen Fähigkeiten, sowohl in Schweizer- als auch in Hochdeutsch. Schliesslich wurden auch die Bedingungen untersucht, welche den Erhalt der Migrationssprache fördern und einen Beitrag für die Lernprozesse in der Schule darstellen.

Schlagworte: Zweisprachigkeit, Erzählfähigkeiten, Spanischer Spracherwerb, Minderheitssprache

\section{Sviluppo di capacità narrative dei bambini bilingui. L'acquisizione dello spagnolo nel contesto Svizzero-tedesco.}

\section{Riassunto}

Il presente contributo analizza le capacità linguistiche dei bambini bilingui spagnolo-svizzero tedesco in occasione della produzione orale di racconti di esperienze personali. Concentrandosi sulla produzione orale in spagnolo prima dell'inizio della scuola elementare, questo studio esplora lo sviluppo precoce delle capacità narrative, considerate un importante precursore dell'alfabetizzazione iniziale dei bambini (Dickinson \& Tabors, 2001). Il nostro studio si basa su esempi di testi prodotti da 24 bambini ripartiti in due gruppi di età, 3 e 5 anni. Analizziamo inoltre un questionario proposto alle famiglie dei bambini, 
che esplora le pratiche linguistiche nell'ambito familiare e la biografia linguistica dei bambini. Lo studio rivela delle differenze tra i due gruppi di età, così come un legame tra le prestazioni discorsive dei bambini e le pratiche linguistiche familiari. Lo studio inoltre permette di identificare alcune capacità in spagnolo che potrebbero potenzialmente favorire le capacità narrative nella lingua quotidianamente parlata, lo svizzero-tedesco, e, in ultima istanza, nel tedesco standard. Infine, questi risultati ci portano a riflettere sulle condizioni che favoriscono la conservazione della lingua della migrazione $\mathrm{d}$ i suoi apporti potenziali alla scolarizzazione.

Parole chiave: Bilinguismo, capacità narrative, acquisizione dello spagnolo, lingua minoritaria

\section{Development of narrative competences from bilingual children: The acquisition of Spanish in the german-speaking part of Switzerland.}

\section{Summary}

The main goal of this study is to examine the oral versions of personal experiences produced by spanish/swiss german bilingual children. This work focuses on the bilingual childrens' capacities before the beginning of primary school, and examines the early development of narrative capacity which is an important precursor to literacy (Dickinson \& Tabors, 2001). A total of 24 narratives produced by 3 and 5 years old children were analysed. A questionnaire was also distributed to the families, in order to know the linguistic practices at home and the childrens' linguistic biographies. Results show developmental differences between the younger and older children. Besides, they also reveal a link between the linguistic practices in the family and the narrative performance. Moreover, some narrative capacities in Spanish that could also be transferred to the majority language are observed. Finally, we reflect on the conditions that promote the preservation of Spanish and its potential contributions to schooling.

Key words: Bilinguisme, narrative competences, Spanish acquisition, minority language. 
